INPLASY

PROTOCOL

To cite: Chang. Ultrasound Imaging for the Assessment of Sarcopenia: A Protocol for An Umbrella Review. Inplasy protocol 2021110028. doi: 10.37766/inplasy2021.11.0028

Received: 08 November 2021

Published: 08 November 2021

Corresponding author:

Ke-Vin Chang

kvchang011@gmail.com

Author Affiliation:

Department of Physical

Medicine and Rehabilitation, National Taiwan University Hospital, Bei-Hu Branch, Taipei, Taiwan.

Support: TSUM.

Review Stage at time of this submission: Preliminary searches.

Conflicts of interest:

None declared.

\section{Ultrasound Imaging for the Assessment of Sarcopenia: A Protocol for An Umbrella Review}

Chang, KV1.

Review question / Objective: To systematically investigate the existing evidence of ultrasound imaging for the diagnosis and evaluation for sarcopenia.

Condition being studied: Sarcopenia.

Information sources: A systemic literature search is conducted in PubMed, Medline, EMBASE, and Web of Science for reviews using a systematic way for literature search and meta-analyses. The reference lists or bibliographies of the available review articles and meta-analyses are scrutinized for additional candidates. Reviews that do not use a systematic way for literature search are excluded from the umbrella review.

INPLASY registration number: This protocol was registered with the International Platform of Registered Systematic Review and Meta-Analysis Protocols (INPLASY) on 08 November 2021 and was last updated on 08 November 2021 (registration number INPLASY2021110028).

\section{INTRODUCTION}

Review question / Objective: To systematically investigate the existing evidence of ultrasound imaging for the diagnosis and evaluation for sarcopenia.

Condition being studied: Sarcopenia.

\section{METHODS}

Search strategy: The combinations of the following keywords are used for literature search, including sarcopenia"," muscle loss", "muscle wasting", "muscle atrophy", "ultrasound", "ultrasonography", "sonoelastograhy", "review", "systematic review" and "meta-analysis". 
Participant or population: Old adults and participants with sarcopenia.

Intervention: Ultrasound imaging.

Comparator: Other imaging methods.

Study designs to be included: Reviews using a systematic way for literature search and meta-analyses.

Eligibility criteria: (1) reviews using a systematic way for literature search and meta-analysis probing the usefulness of ultrasound in evaluation of skeletal muscles for sarcopenia (2) enrollment of at least one study investigating patients with sarcopenia, (3) reporting any of the ultrasound indicators and (4) targeting the geriatric population (age $\geq 65$ years).

Information sources: A systemic literature search is conducted in PubMed, Medline, EMBASE, and Web of Science for reviews using a systematic way for literature search and meta-analyses. The reference lists or bibliographies of the available review articles and meta-analyses are scrutinized for additional candidates. Reviews that do not use a systematic way for literature search are excluded from the umbrella review.

Main outcome(s): The results will be reported based on the types of ultrasound modes (B mode vs. sonoelastography), sites of applications, reliability/validity of ultrasound measurements of muscle mass and the capability of ultrasound indicators for differentiating sarcopenia.

Quality assessment / Risk of bias analysis: The quality of the included articles will be assessed by using the AMSTAR 2 (A Measurement Tool to Assess Systematic Reviews) critical appraisal tool independently by the two authors.

Strategy of data synthesis: The extracted data will be narrated at the level of systematic reviews and meta-analyses.
Sensitivity analysis: Not applicable.

Country(ies) involved: Taiwan.

Keywords: Sarcopenia, muscle loss, ultrasound, sonography, elastography.

Contributions of each author:

Author 1 - Ke-Vin Chang.

Subgroup analysis: Not applicable. 